\title{
Laboratory- and field-based testing as predictors of skating performance in competitive-level female ice hockey
}

This article was published in the following Dove Press journal:

Open Access Journal of Sports Medicine

18 August 2016

Number of times this article has been viewed

\author{
Tommy Henriksson ${ }^{1,2}$ \\ Jason D Vescovi ${ }^{3}$ \\ Anncristine Fjellman- \\ Wiklund $^{4}$ \\ Kajsa Gilenstam' \\ 'Sport Medicine Unit, Department \\ of Community Medicine and \\ Rehabilitation, ${ }^{2}$ The National \\ Graduate School of Gender Studies, \\ Umeå University, Umeå, Sweden; \\ ${ }^{3}$ Faculty of Kinesiology and Physical \\ Education, University of Toronto, \\ Toronto, ON, Canada; ${ }^{4}$ Physiotherapy \\ Unit, Department of Community \\ Medicine and Rehabilitation, Umeå \\ University, Umeå, Sweden
}

Objectives: The purpose of this study was to examine whether field-based and/or laboratorybased assessments are valid tools for predicting key performance characteristics of skating in competitive-level female hockey players.

Design: Cross-sectional study.

Methods: Twenty-three female ice hockey players aged 15-25 years (body mass: $66.1 \pm 6.3 \mathrm{~kg}$; height: $169.5 \pm 5.5 \mathrm{~cm}$ ), with $10.6 \pm 3.2$ years playing experience volunteered to participate in the study. The field-based assessments included $20 \mathrm{~m}$ sprint, squat jump, countermovement jump, 30-second repeated jump test, standing long jump, single-leg standing long jump, $20 \mathrm{~m}$ shuttle run test, isometric leg pull, one-repetition maximum bench press, and one-repetition maximum squats. The laboratory-based assessments included body composition (dual energy X-ray absorptiometry), maximal aerobic power, and isokinetic strength (Biodex). The on-ice tests included agility cornering s-turn, cone agility skate, transition agility skate, and modified repeat skate sprint. Data were analyzed using stepwise multivariate linear regression analysis. Linear regression analysis was used to establish the relationship between key performance characteristics of skating and the predictor variables.

Results: Regression models (adj $R^{2}$ ) for the on-ice variables ranged from 0.244 to 0.663 for the field-based assessments and from 0.136 to 0.420 for the laboratory-based assessments. Singleleg tests were the strongest predictors for key performance characteristics of skating. Single leg standing long jump alone explained $57.1 \%, 38.1 \%$, and $29.1 \%$ of the variance in skating time during transition agility skate, agility cornering s-turn, and modified repeat skate sprint, respectively. Isokinetic peak torque in the quadriceps at $90^{\circ}$ explained $42.0 \%$ and $32.2 \%$ of the variance in skating time during agility cornering s-turn and modified repeat skate sprint, respectively.

Conclusion: Field-based assessments, particularly single-leg tests, are an adequate substitute to more expensive and time-consuming laboratory assessments if the purpose is to gain knowledge about key performance characteristics of skating.

Keywords: exercise physiology, test methodology, sport, physiological characteristics

\section{Introduction}

Ice hockey is a high-intensity intermittent contact sport with high physical demands of the neuromuscular and cardiovascular systems. ${ }^{1}$ Performance in ice hockey is determined through an interaction of several factors, which poses challenges to capture all aspects that could potentially impact the ability to compete at a high level. ${ }^{2}$ The assessment of physical and physiological attributes is considered a valuable component for monitoring player development, talent identification, as well as prediction of specific performance variables, such as skating. ${ }^{3-5}$ So far, most research regarding
Correspondence: Tommy Henriksson Sports Medicine Unit, Department of Community Medicine and Rehabilitation, Umeå University, Gösta Skoglunds väg 3, Umeå SE-90187, Sweden

Tel +4690786 66 II

Email tommy.henriksson@umu.se 
physiological factors associated with skating performance are based on males. ${ }^{6}$ Research on female hockey, and particularly competitive-level female hockey, is somewhat limited. ${ }^{5}$ However, because of different biological, sociocultural, and financial preconditions, knowledge of male hockey cannot outright be applied on female hockey. ${ }^{7}$ Thus, while skating is considered the most important skill in ice hockey, ${ }^{8}$ factors that might best explain key performance characteristics of skating in competitive-level female ice hockey remains largely unknown. Consequently, it is also currently unknown whether laboratory- or field-based tests are best suited for assessing key performance characteristics of skating in competitivelevel female ice hockey. ${ }^{4,9,10}$

Laboratory-based assessments are performed under wellcontrolled environments, provide highly accurate outcomes, and might replicate the physiological demands of ice hockey; however, they require expensive equipment, expertise, are time-consuming, and lack movement specificity ${ }^{11}$ in comparison to field-based assessments. ${ }^{12}$ Some researchers have indicated that field-based testing is a valid predictor for successful performance in male ice hockey, ${ }^{10,13}$ while others considered field-based testing as proxies for specific components of hockey performance. ${ }^{14,15}$ This is a relatively unexplored area in female ice hockey; however, one recent study ${ }^{16}$ has indicated that field-based assessments can be used to predict speed and recovery ability in lower level (division III) female ice hockey. Currently, no study has compared the predictive value of physiological and physical outcomes from laboratory- and field-based protocols to key performance characteristics of skating.

Thus, the purpose of this study was to examine whether field-based and/or laboratory-based assessments are valid tools for predicting key performance characteristics of skating in competitive-level female hockey players. In accordance with the principle of specificity, exercise adaptions are specific to the mode and intensity of training. ${ }^{17}$ As much of the time for a skater is spent on one leg, ${ }^{18}$ our hypothesis is that single-leg exercises are more sport-specific for a hockey player and will thus provide more useful information regarding key performance characteristics of skating than two-leg exercises.

\section{Methods}

This study had a cross-sectional design, and the protocol was reviewed and approved by the Regional Ethical Review Board at Umeå University, Department of Medical Research (Dnr: 2012-22-31M). Experimental procedures and study risks were verbally described, and written informed consent was obtained from the participants (and parents when appropriate) prior to volunteering in the study. Prior to testing, participants completed a questionnaire regarding general health status, assuring they were not sick, injured, or using any medication that could affect their health or performance. The participants were instructed to refrain from food and caffeine within 3 hours of testing. Twenty-three female ice hockey players volunteered for the study (Table 1). Athletes were recruited from a single team participating in the highest league (Riksserien) in Sweden. Participants had 10.6 \pm 3.2 years of playing experience, typically trained 9-10 hours, and competed in two games per week.

The participants were tested pre- $(n=18)$ or postseason $(\mathrm{n}=5)$. Inter- and intraday testing order was standardized (Table 2). Physiological and performance assessments were

Table I Participant characteristics $(\mathrm{n}=23)$

\begin{tabular}{lll}
\hline Variable & Mean \pm SD & Range \\
\hline Age (years) & $18.04 \pm 2.14$ & $15.00-25.00$ \\
Body mass $(\mathrm{kg})$ & $66.11 \pm 6.27$ & $56.90-79.70$ \\
Body height $(\mathrm{cm})$ & $169.45 \pm 5.50$ & $158.70-179.50$ \\
DXA body fat $(\%)$ & $25.57 \pm 4.73$ & $17.00-34.50$ \\
DXA lean body mass $(\mathrm{kg})$ & $46.67 \pm 4.23$ & $37.11-53.38$ \\
\hline
\end{tabular}

Abbreviations: DXA, dual X-ray absorptiometry; SD, standard deviation.

Table 2 Testing schedule: inter- and intraday testing order

\begin{tabular}{llll}
\hline Field-based assessments & & On-ice tests & Laboratory-based assessments \\
\hline Day I & Day 2 & Day 3 & Day 4 \\
\hline Anthropometrics & IRM squat & Agility cornering s-turn & DXA \\
20 m sprint & IRM bench press & Cone agility skate & Maximal aerobic power \\
Squat jump & Isometric leg pull & Transition agility skate & Isokinetic strength \\
Countermovement jump & & MRSS & \\
Bosco 30-second repeated jump test & & & \\
Standing long jump & & & \\
Single-leg standing long jump & & & \\
20 m shuttle run & & & \\
\hline
\end{tabular}

Notes: Days I-2 were performed off-ice, day 3 on-ice, and day 4 in laboratory. Days 1-3 were performed during 3 consecutive days, followed by day 4 approximately I-2 weeks later. At least 5 minutes of recovery was provided between trials to minimize fatigue.

Abbreviations: DXA, dual X-ray absorptiometry; MRSS, modified repeat skate sprint; IRM, one-repetition maximum. 
Table 3 Test data $(n=23)$

\begin{tabular}{|c|c|c|}
\hline Description & Mean \pm SD & Range \\
\hline \multicolumn{3}{|l|}{ Field-based assessments } \\
\hline Isometric leg pull (kg) & $111.25 \pm 14.28$ & $89.50-139.70$ \\
\hline Isometric leg pull (kg/kg body mass) & $1.65 \pm 0.26$ & $1.20-2.10$ \\
\hline Squat - IRM (kg) & $89.79 \pm 14.17$ & $60.00-115.00$ \\
\hline Squat - IRM (kg/kg body mass) & $1.37 \pm 0.19$ & $1.01-1.70$ \\
\hline Bench press - IRM (kg) & $47.03 \pm 6.70$ & $35.00-60.00$ \\
\hline Bench press - IRM ( $\mathrm{kg} / \mathrm{kg}$ body mass) & $0.72 \pm 0.09$ & $0.50-0.89$ \\
\hline Beep test $(\mathrm{km})$ & $1.70 \pm 2.67$ & $1.04-2.14$ \\
\hline Bosco test fatigue (\%) & $0.74 \pm 0.10$ & $0.58-1.01$ \\
\hline Standing long jump (m) & $2.03 \pm 0.16$ & $\mathrm{I} .7 \mathrm{I}-2.39$ \\
\hline Standing long jump right (m) & $1.7 I \pm 0.15$ & $1.33-1.98$ \\
\hline Squat jump $(\mathrm{cm})$ & $26.04 \pm 3.82$ & $19.30-33.60$ \\
\hline Countermovement jump (cm) & $28.02 \pm 5.13$ & $16.72-37.80$ \\
\hline Sprint $20 \mathrm{~m}(\mathrm{~s})$ & $3.50 \pm 0.18$ & $3.21-3.98$ \\
\hline \multicolumn{3}{|l|}{ Laboratory-based assessments } \\
\hline Biodex quadriceps $90 \%$ s peak torque $(\mathrm{N} \cdot \mathrm{m})$ & $142.12 \pm 21.03$ & $96.80-176.90$ \\
\hline Biodex quadriceps $90 \%$ s peak torque/bw (\%) & $217.02 \pm 33.62$ & $151.80-268.00$ \\
\hline Biodex quadriceps $90^{\circ} / \mathrm{s}$ time to peak (MSEK) & $416.09 \pm 104.48$ & $190.00-590.00$ \\
\hline Biodex quadriceps $90 \%$ s total work (J) & $802.55 \pm 124.95$ & $625.60-1,108.80$ \\
\hline Biodex quadriceps $210 \%$ s peak torque $(\mathrm{N} \cdot \mathrm{m})$ & $106.25 \pm 17.08$ & $84.60-148.20$ \\
\hline Biodex quadriceps $210 \%$ s peak torque/bw (\%) & $162.54 \pm 28.98$ & $|2| .80-24 \mid .80$ \\
\hline Biodex quadriceps $210^{\circ} / \mathrm{s}$ time to peak (MSEK) & $219.13 \pm 91.70$ & $110.00-480.00$ \\
\hline Biodex quadriceps $210 \%$ s total work (J) & $1,022.39 \pm 148.88$ & $778.40-1,384.50$ \\
\hline Ergo meter cycle test (mL/kg/min) & $47.24 \pm 6.16$ & $37.90-59.20$ \\
\hline Ergo meter cycle test (L/min) & $3.11 \pm 0.42$ & $2.30-4.00$ \\
\hline \multicolumn{3}{|l|}{ On-ice performance variables } \\
\hline MRSS total time (s) & $62.28 \pm 3.81$ & $55.81-72.09$ \\
\hline Transition agility average $(s)$ & $11.93 \pm 0.89$ & $10.73-14.10$ \\
\hline Cone agility average (s) & $9.47 \pm 0.47$ & $8.53-10.55$ \\
\hline S-cornering average (s) & $9.6 \pm 0.28$ & $8.88-10.23$ \\
\hline
\end{tabular}

Note: All Biodex measurements represent right-side values.

Abbreviations: min, minutes; MRSS, modified repeat skate sprint; s, seconds; IRM, one-repetition maximum; SD, standard deviation.

performed during 4 days through field-based, laboratorybased, and on-ice tests (Table 3). Days 1-3 were performed during 3 consecutive days in the team's own premises, followed by day 4 approximately $1-2$ weeks later at Umea University's sport-testing facility. The interval between laboratory and field tests was required because of traveling distance to our laboratory and in consideration to the team's own activities. Prior to any physical testing, the participants performed a standardized warm-up of $\sim 10$ minutes, including exercises such as jogging, shuffling, sprinting, skating, and dynamic stretching exercises. Prior to each performance, test procedures were thoroughly introduced and demonstrated so the participants were familiar with the criteria for correct execution. Furthermore, the participants were allowed to some test trials before each of the actual tests.

Day 1 was performed in the gym. Body mass was measured to the nearest kilogram $(\mathrm{kg})$ with a digital scale (Fitbit Aria, WS-30; Fitbit Inc., San Francisco, CA, USA). Height was measured to the nearest $0.5 \mathrm{~cm}$ with the participant standing against a flat wall and a rectangular object placed concurrently against both the subject's head and the wall. ${ }^{19}$

Timing gates (ChronoJump Co., Barcelona, Spain; Version 1.3.9.0) were used for the $20 \mathrm{~m}$ sprint. Participants began the test from a stationary sprint start with one foot in line with the laser. Participants were allowed a voluntary start and received two trials with at least 2 minutes of recovery in between trials. The best score was recorded to the nearest $0.01 \mathrm{~s}$.

The countermovement and squat jump tests as well as the 30-second repeated jump tests were performed as described by $\mathrm{Bosco}^{20}$ using a Chronojump (ChronoJump Co.; Version 1.3.9.0). For the countermovement and squat jump tests, each subject received three trials with at least 60 seconds of rest in-between jumps. The best of three trials was recorded to the nearest $0.1 \mathrm{~cm}$. For the 30 -second repeated jump tests, the height of every jump was recorded to the nearest $0.1 \mathrm{~cm}$. Dropoff percent was calculated and recorded to the nearest $0.1 \%$. 
The standing long jump was performed with toes behind the start line; participants performed a countermovement and jumped as far forward as possible. Participants were provided with at least 60 seconds of rest between attempts. Distance was measured to the back edge of the nearest heel, and the best of three trials was recorded to the nearest $1 \mathrm{~cm}$. Single-leg standing long jumps were performed following the same procedures. Participants took off from a single leg but were instructed to land on both feet to reduce the risk of injury.

The $20 \mathrm{~m}$ shuttle run (Beep test) was performed according to the procedure of Léger and Lambert. ${ }^{21}$ Briefly, participants ran back-and-forth on a $20 \mathrm{~m}$ course being paced by audible beeps, which become progressively faster. When participants failed to reach two consecutive end lines on time, the test was terminated and the total completed distance (m) was recorded as the result.

Day 2 was performed in the gym. The one-repetition maximum (1RM) back squat and 1RM bench press were performed following the procedures described by Fleck and Kreamer. ${ }^{22}$ For a successful squat attempt, participants must lower until their hamstrings/thighs were parallel to the floor. For a successful bench press attempt, participants must lower the bar until it touches the chest, while retaining the feet, buttocks, and shoulders in contact with the surface. Participants were instructed to reach 1RM within four to five attempts to avoid fatigue; $2-4$ minutes of rest between the sets were mandatory. The maximal approved load was registered as their result.

Isokinetic leg pull was performed using a strength dynamometer (Vetek AB, Vaddo, Sweden; VZ101BH 500 kg). The participants were instructed to stand upright on the base of the dynamometer with the feet shoulder width apart, arms hanging straight down to hold the center of the bar with both hands, palms facing the body. The chain was adjusted so that the bar was set just below the kneecaps. In this position, back slightly bent, forward at the hips, head held upright, the participants were instructed to pull as hard as possible and try to straighten their legs without bending their back. No jerky movements were allowed. Participants received two trials with 2-3 minutes rest in between, and the maximum force was recorded to the nearest $0.1 \mathrm{~kg}$.

Day 3 was performed on-ice. Participants were tested wearing full equipment, including their stick.

The agility cornering s-turn test was modified from the protocol of Gilenstam et $\mathrm{al}^{7}$ by reducing the distance between goal lines to $17.7 \mathrm{~m}$. Participants began behind the goal, completed an s-shaped pattern around the face-off circles, and finished by the nearest blue line (Figure 1A).

Participants started at the blue line for the cone agility test, skated as quickly as possible around three pylons situated on the center red line (two cones) and blue line (one cone), and finished back over the blue line (Figure 1B). ${ }^{15}$

The transition agility test followed the protocol of the National Skills Standards and Testing Program published by Hockey Canada. ${ }^{23}$ The participants started on the line at the bottom of circle, skated forward to far right-side pylon, pivoted, and skated backward to lower side pylon. The participants then pivoted and skated forward to left-side pylon, pivoted, and skated backward to lower left pylon. The participants then pivot and skate forward over the far goal line at the far end of the circle (Figure 1C).

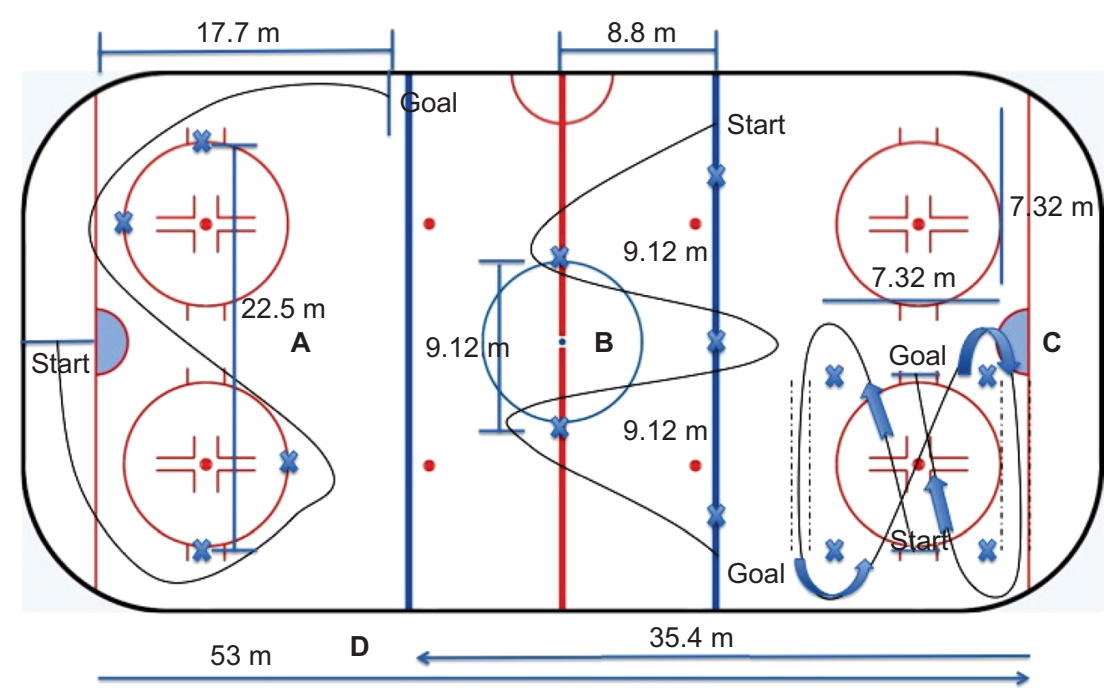

Figure I Description of on-ice tests.

Notes: A: Agility cornering s-turn, B: cone agility, C: transition agility, D: modified repeat skate sprint. 
The modified repeat skate sprint (MRSS) test was modified from the one described by Bracko and George ${ }^{3}$ by having the athletes skate four repetitions of $88.4 \mathrm{~m}$ on a 30 -second clock. Participants skated from one end line to the other $(53 \mathrm{~m})$ and then returned to the initial blue line $(35.4 \mathrm{~m})$. After each repetition, the athletes had the remainder of 30 seconds for recovery before the next repetition was initiated (Figure 1D). Participants received two trials on each test (except for the MRSS). The direction was reversed for the second trial. Dual hand-timed stopwatches were used to record the time. The average between the two trials was recorded to the nearest 0.01 second.

Day 4 was performed in the laboratory. Body composition was assessed via Lunar iDXA (GE Medical Systems Lunar, WI, USA; Encore Version 14.10.022). DXA is considered a reliable method for clinical assessment of body composition. $^{24}$

Maximal aerobic fitness was assessed via direct gas analysis using a ramp protocol, which was applied using an electronically braked cycle ergometer (Monark 839E) to volitional fatigue. The initial intensity was $40 \mathrm{~W}$ with $40 \mathrm{~W}$ increments every 3 minutes up to the attainment of a blood lactate concentration of $4 \mathrm{mmol} / \mathrm{L}$, at which point intensity was increased $20 \mathrm{~W}$ every 30 seconds to volitional fatigue. The participants were asked to pedal at $70-80 \mathrm{rpm}$ for the duration of the test. Expired respiratory gases were measured continuously using a Jaeger Oxycon Pro system Version 5.3 (Viasys Healthcare, Conshohocken, PA, USA). Values for $\dot{\mathrm{V}} \mathrm{O}_{2}$ were averaged over 20 seconds with the highest value recorded as $\dot{\mathrm{VO}}_{2}$ peak. An indwelling catheter was used to sample venous blood following each stage and analyzed for blood lactate concentration (YSI 2300 STAT PLUS; Yellow Springs International, Yellow Springs, OH, USA).

Participants then recovered for 4 hours before isokinetic strength was assessed through a calibrated isokinetic dynamometer for the knee flexors and extensors (Biodex System 4; Biodex Co., New York, NY, USA) as illustrated by Gilenstam et $\mathrm{al}^{7}$ each leg assessed in random order. Following some test-specific warm-up repetitions in the dynamometer, participants were given a 2-minute recovery period before performing the test. Participants performed five maximal concentric (utilizing quadriceps and hamstrings) contractions in the knee joint, at the angular velocity of $90^{\circ} / \mathrm{s}$, and ten maximal contractions at $210 \%$, separated by a 2 -minute recovery period. Peak torque $/ \mathrm{kg}$ body mass and total work were recorded at both speeds.

Data were analyzed by using SPSS 22.0 (IBM Corporation, Armonk, NY, USA).
Data were analyzed for patterns of missing values, which showed that much information would likely be lost if simple list-wise deletion was used. Little' ${ }^{25}$ missing completely at random test failed to reject the null hypothesis, which indicated that missing values were completely random. Missing values confirmed as random allowed data transformation through maximum likelihood estimation. The method uses all available data to identify the parameter values with the highest probability of producing the sample data and replace missing values. ${ }^{26}$ Data were tested for normality through Shapiro-Wilks test, $P>0.05$, which indicated normality. Pearson's product-moment correlation was used to assess relationships between right and left leg values in all single-leg exercises. The analysis showed right and left leg values to be significantly correlated $(r=0.586-0.950$, $P<0.05)$; therefore, in order to limit the number of variables in the analysis, all left leg values were excluded. Stepwise multiple linear regression analyses were performed until the strongest laboratory- and field-based model could be identified for each of the four on-ice variables. All models were adjusted for degrees of freedom. Adjusted $R^{2}$ was used because it is a somewhat more conservative measure than the $R^{2}$ value.

\section{Results}

The adjusted coefficient of determination (adj $R^{2}$ ) of the regression models ranged from 0.244 to 0.663 for the fieldbased assessments and from 0.136 to 0.420 for the laboratorybased assessments. The strongest regression models from the combination of field- and laboratory-based variables, respectively, are reported later.

\section{Transition agility}

Laboratory-based prediction model (adj $R^{2}=0.264, P=0.007$ ) $=(-0.004 \times$ Biodex hamstrings $210 \%$ s total work $)-14.705$. Field-based prediction model $\left(\operatorname{adj} R^{2}=0.244, P=0.010\right)=$ $(-4.594 \times$ single-leg standing long jump $)-19.798$.

\section{Cone agility}

Laboratory-based prediction model (adj $R^{2}=0.136, P=0.047$ ) $=\left(-0.009 \times\right.$ Biodex quadriceps $90^{\circ} / \mathrm{s}$ peak $)-10.804$. Field based prediction model $\left(\operatorname{adj} R^{2}=0.244, P=0.010\right)=(-2.558$ $\times$ Bosco test fatigue) -11.36 .

\section{Agility cornering s-turn}

Laboratory-based prediction model (adj $R^{2}=0.446, P<0.001$ ) $=(-0.009 \times$ Biodex quadriceps $90 \%$ s peak $)-10.877$. Field-based prediction model $\left(\operatorname{adj} R^{2}=0.244, P=0.010\right)$ 
$=(-1.168 \times$ single-leg standing long jump $)+(-0.02 \times$ body mass $)+(-0.338 \times$ Beep test $)+(-0.919 \times$ Bosco test fatigue) -14.142 .

\section{Modified repeated skate sprint}

Laboratory-based prediction model $\left(\operatorname{adj} R^{2}=0.322, P=0.003\right)$ $=(-0.21 \times$ Biodex hamstrings $90 \%$ s right peak $)-78.115$. Field-based prediction model $\left(\right.$ adj $\left.R^{2}=0.540, P<0.001\right)=$ $(-12.353 \times$ single-leg standing long jump $)+(-0.305 \times$ body height $)+(-0.174 \times$ bench press $1 \mathrm{RM})-143.317$.

Predictor variables for all on-ice variables are presented in Tables 4 and 5. Single tests often produced stronger predictions than a combination of tests, whereas single-leg tests were the strongest predictors for skating performance. Single-leg standing long jump explained $57.1 \%, 38.1 \%$, and $29.1 \%$ of the variance in skating time during transition agility skate, agility cornering s-turn, and MRSS, respectively. Isokinetic peak torque of the quadriceps at $90 \% \mathrm{~s}$ explained $42.0 \%$ and $32.2 \%$ of the variance in skating time during agility cornering s-turn and MRSS, respectively. Isokinetic total work of the hamstrings at $210 \%$ s explained $26.4 \%$, and

Table 4 Field-based predictor variables for all on-ice performance regression models $(n=23)$

\begin{tabular}{lllll}
\hline On-ice & Predictor variable(s) & $\beta$ & $t$ & $P$ \\
\hline Transition agility & Single-leg standing long & -0.769 & -5.506 & $<0.001$ \\
& jump & & & \\
Cone agility & $\begin{array}{l}\text { Bosco 30-second repeated } \\
\text { jump test }\end{array}$ & -0.528 & -2.847 & 0.010 \\
Agility cornering & Single-leg standing long & -0.615 & -4.765 & $<0.001$ \\
s-turn & jump & & & \\
& Body mass & -0.433 & -3.358 & 0.003 \\
& Beep test & -0.317 & -2.489 & 0.023 \\
& Bosco test & -0.314 & -2.489 & 0.024 \\
MRSS & $\begin{array}{l}\text { Single-leg standing long } \\
\text { jump }\end{array}$ & -0.485 & -3.307 & $<0.001$ \\
& Body height & $-0.44 I$ & -3.017 & 0.004 \\
& Bench press & -0.305 & -2.106 & 0.049 \\
\hline
\end{tabular}

Note: Alpha level was set at 0.05 to determine statistical significance.

Abbreviation: MRSS, modified repeat skate sprint.

Table 5 Laboratory-based predictor variables for the on-ice performance regression models $(n=23)$

\begin{tabular}{|c|c|c|c|c|}
\hline On-ice & Predi & $\beta$ & $t$ & $P$ \\
\hline \multirow[t]{2}{*}{ Transition agility } & $\mathrm{Bi}$ & -0.545 & $-2.98 \mid$ & 0.007 \\
\hline & $210 \%$ & & & \\
\hline Cone & eps $90 \%$ s peak & -0.418 & -2.109 & 0.049 \\
\hline Agility cornering & Biode & & & \\
\hline s-turn & $90 \%$ s peak torque & -0.668 & -4.115 & $<0.001$ \\
\hline MRSS & Biodex hamstrings $90 \%$ s peak & -0.594 & -3.386 & 0.003 \\
\hline
\end{tabular}

Note: Alpha level was set at 0.05 to determine statistical significance.

Abbreviations: MRSS, modified repeat skate sprint; s, seconds. peak torque of the hamstrings at $90 \%$ s explained $13.6 \%$ in skating time during transition agility skate and cone agility skate, respectively.

\section{Discussion}

The findings from this study indicate that field-based assessments, and particularly single-leg exercises, provide a valid method for coaches to gain knowledge about key performance characteristics for female competitive ice hockey players. Our results showed stronger predictive power for field-based assessments compared to laboratory-based assessments, even if both methods produced valid predictive models for the included skating tests. Further, our results showed that single-leg tests produced strong predictive models for long crossover cornering ability, skating transitions (forward-tobackward pivoting), and skating endurance.

Compared to laboratory-based assessments, the fieldbased assessments produced stronger predictions for all four skating tests. However, the laboratory-based assessments also proved acceptable indicators of the outcome for the same set of skating tests. This indicates that both field-based and laboratory-based assessments can be considered valid tools for coaches when they want to gain knowledge about a female player's skating abilities. These findings are in agreement with previous studies on male hockey players, ${ }^{14,15,27}$ which have shown correlations between skating performance and field-based assessments. Farlinger et $\mathrm{al}^{14}$ reported that on-ice cornering and sprint ability were strongly correlated to three hop jump ( $r=-0.53, r=-0.78)$ and broad jump $(r=-0.59$, $r=-0.74)$. This finding is consistent with our results, where single-leg standing long jump was the strongest predictor for agility cornering s-turn, MRSS, and transition agility. Furthermore, the predictive models for agility cornering s-turn and MRSS include negative coefficient for body mass and body height, which likely reflect the importance of relative strength and power in the lower body to skating performance in ice hockey. This conclusion is supported by the findings from Gilenstam et al, ${ }^{7}$ who argued that body composition was related to skating speed and acceleration in female ice hockey players. They found positive correlations between skating time, acceleration and speed, and body weight $(r=0.639$, $P=0.034$ and $r=0.831, P=0.002$, respectively). They also found a negative correlation between skating time, speed, and lean body mass $(r=0.773, P=0.005)$.

Furthermore, Farlinger et a $\mathrm{a}^{14}$ reported significant correlations between maximum number of push-ups to both on-ice sprints $(r=-0.60)$ and on-ice cornering ability $(r=-0.38)$. However, as far as we know, the impact of maximal force production in the upper body to skating performance has 
not previously been investigated. Thus, it is interesting that $1 \mathrm{RM}$ bench press contributed to the predictive model for MRSS. Previous studies on running ${ }^{28-30}$ have indicated that the arm swing serves as an important mechanism in maximum velocity sprints, both in order to enhance propulsive forces but also to counterbalance the rotary momentum of the legs. Hence, it is logical that greater strength in the upper body could contribute to an increased speed in linear forward skating as well.

The second central finding was the strong predictive nature of single-leg tests as indicators for the included on-ice skating tests. We found that this result applied to both fieldbased assessments, using a single-leg standing long jump test, and laboratory-based assessments measuring isokinetic strength in both the quadriceps and the hamstrings. This finding seems logical since quadriceps (and gluteus maximus) are considered the major contributing muscles in skating, which is the same as those in single-leg standing long jump and the isokinetic quadriceps test. ${ }^{2}$ The hamstrings are not previously believed to contribute directly to the power production in skating but are known to be important stabilizers for the knee in the sliding phase of a stride. ${ }^{2}$ Thus, the hamstrings support the quadriceps during force generation, which might explain why isokinetic strength in the hamstrings was a strong predictor of skating performance. This conclusion is in accordance with Bracko, ${ }^{18}$ who reported that a skater who is powerful, in relation to body weight, primarily in quadriceps and hamstrings are able to skate faster and maintain a higher work output during her time on the ice.

Furthermore, it seems reasonable that single-leg exercises produced the strongest predictive models for skating performance, since a predominant amount of time $(\sim 80 \%)$ spent during forward skating is carried out in single-support phase. $^{2}$ In addition, Fortier et $\mathrm{al}^{31}$ reported that a player has to withstand greater forces on the supporting leg, even when both skates are in contact with the ice, for example, the outer leg is generally subjected to greater forces during a turn compared to the inner leg. This underlines the importance for an ice hockey player to develop and resist force in singleleg support phase. Thus, together with previous research, our findings indicate that the strength and power a player can produce in single-leg support may be a limiting factor in how well a female player can carry out technical skating elements in high speed.

\section{Limitations}

There are several limitations to this study that should be considered. First, data collection occurred before the season for some participants and following the season for others. It is plausible that performance tests could be impacted by when these tests were performed; however, a comparison between these individuals revealed no significant differences in any of the dependent variables. Thus, we are confident in the outcomes of the regression models. Second, no agility exercises were included in the study, which can be considered a limitation. However, the concept of agility includes physical change of direction speed (CODS) as well as perceptual cognitive ability to react to a stimulus. ${ }^{32}$ Thus, is seemed reasonable to only include tests that measure CODS and physical components of CODS, since the purpose of the study was to investigate skating performance and not game performance. A third limitation could be the use of handheld stop watches instead of electronic timing devices. We consider the effect of this limitation to be minimized by the use of average value from multiple timers.

\section{Conclusion}

Our findings suggest that off-ice tests, and particularly singleleg tests, prove a valid method for coaches to gain knowledge about performance characteristics for skating in competitive female hockey players. Single-leg standing long jump was found to produce the strongest predictive models for long crossover cornering ability, skating transitions (forward-tobackward pivoting), and skating endurance. Our findings also showed that field-based assessments could be an adequate substitute to laboratory-based assessments if the purpose is to gain knowledge about key performance characteristics for skating. Future research would need to investigate if this knowledge is applicable to other standards (eg, developmental and elite) of female hockey players.

\section{Practical application}

As a coach, time and resources are often limited. Therefore, practice has to be appropriate to the purpose and evaluated in relation to actual performance. This study demonstrates that laboratory-based and field-based assessments can be used to gain knowledge about key performance characteristics for skating, which is considered a vital aspect of ice hockey. ${ }^{12}$ However, field-based assessments give a greater value for the user, being less expensive and more accessible for teams with limited resources. Thus, we recommend including single-leg standing long jump in an overall evaluation of a hockey player since it was the strongest predictor. Notably, the results of this study apply only to testing. Whether it is beneficial to use single-legged exercises to improve skating performance remains to be investigated. 


\section{Acknowledgments}

The authors would like to thank the participants and their coaches for their cooperation and enthusiasm in the study. They would also like to thank Rookie Westin for his expertise and assistance in the data collection. The study was funded by The National Graduate School of Gender Studies, Umeå University, Umeå, Sweden, the Anna Cederberg Foundation, and SJCKMS Kempe Foundation. The study was conducted at the Department of Community Medicine and Rehabilitation, Sport Medicine Unit, Umeå University, Umeå, Sweden.

\section{Disclosure}

The authors report no conflicts of interest in this work.

\section{References}

1. Montgomery DL. Physiology of ice hockey. Sports Med. 1988;5(2): 99-126.

2. Pearsall DJ, Turcotte RA, Murphy SD. Biomechanics of ice hockey. In: Garret WE, Kirkendall DT, editors. Exercise and Sport Science. Philadelphia, PA: Lippincott Williams \& Wilkins; 2000:675-692.

3. Bracko MR, George JD. Prediction of ice skating performance with off-ice testing in women's ice hockey players. $J$ Strength Cond Res. 2001;15(1):116-122.

4. Green MR, Pivarnik JM, Carrier DP, Womack CJ. Relationship between physiological profiles and on-ice performance of a national collegiate athletic association division I hockey team. J Strength Cond Res. 2006;20(1):43-46.

5. Ransdell LB, Murray TM, Gao Y. Off-ice fitness of elite female ice hockey players by team success, age and player position. $J$ Strength Cond Res. 2013;27(4):875-884.

6. Pelak CF. Women's collective identity formation in sports: a case study from women's ice hockey. Gend Soc. 2002;16(1):93-114.

7. Gilenstam K, Thorsen K, Henriksson-Larsén KB. Physiological correlates of skating performance in women's and men's ice hockey. $J$ Strength Cond Res. 2011;25(8):2133-2142.

8. Montgomery DL. Physiology of ice hockey. In: Garret WE, Kirkendall DT, editors. Exercise and Sport Science. Philadelphia, PA: Lippincott Williams \& Wilkins; 2000:815-828.

9. Potteiger JA, Smith DL, Maier ML, et al. Relationship between body composition, leg strength, anaerobic power, and on-ice skating performance in division I men's hockey athletes. $J$ Strength Cond Res. 2010;24(7):1755-1762.

10. Tarter BC, Kirisci L, Tarter RE, et al. Use of aggregate fitness indications to predict transition into the national hockey league. $J$ Strength Cond Res. 2009;23(6):1828-1832.

11. Durocher JJ, Guisfredi AJ, Leetun DT, Carter JR. Comparison of on-ice and off-ice graded exercise testing in collegiate hockey players. Appl Physiol Nutr Metab. 2010;35(1):35-39.
12. Bracko MR. On-ice performance characteristics of elite and nonelite women's ice hockey players. J Strength Cond Res. 2001;15(1): $42-47$.

13. Burr JF, Jamnik VK, Dogra S, Gledhill N. Evaluation of jump protocols to assess leg power and predict hockey playing potential. $J$ Strength Cond Res. 2007;21(4):1139-1145.

14. Farlinger CM, Kruisselbrink DL, Fowles JR. Relationship to skating performance in competitive hockey players. $J$ Strength Cond Res. 2007;21(3):915-922.

15. Behm DG, Wahl MJ, Button DC, Power KE, Anderson KG. Relationship between hockey skating speed and selected performance measures. $J$ Strength Cond Res. 2005;19(2):326-331.

16. Janot JM, Beltz NM, Dalleck LD. Multiple variables predict on-ice skating performance in male and female division III ice hockey players. J Sports Sci Med. 2015;14(3):522-529.

17. Kenney WL, Wilmore JH, Costill DL. Physiology of Sport and Exercise. Fifth ed. Champaign: Human Kinetics; 2012.

18. Bracko MR. Biomechanics powers ice hockey performance. Biomechanics. 2004;9:47-53.

19. International Society for the Advancement of Kinanthropometry (ISAK). International Standards for Anthropometric Assessment. Underdale: ISAK; 2001.

20. Bosco C. Strength Assessment with the Bosco's Test. Rome: Italian Society of Sports Science; 1999.

21. Léger LA, Lambert J. A maximal multistage $20 \mathrm{~m}$ shuttle run test to predict $\mathrm{VO}_{2 \max }$. Eur J Appl Physiol. 1982;49(1):1-5.

22. Fleck SJ, Kreamer WJ. Designing Resistance Training Programs. Third ed. Champaign: Human Kinetics; 2004.

23. Hockey Canada [webpage on the Internet]. Handbook for National Skills Standard \& Testing Program. 1999. Available from: http://cdn.agilitycms.com/hockey-canada/Hockey-Programs/Players/Skills-Testing/ Downloads/nsst_handbook_e.pdf. Accessed September 11, 2015.

24. Ackland TR, Lohman TG, Sundgot-Borgen J, et al. Current status of body composition assessment in sport. Sports Med. 2012;42(3): 227-249.

25. Little RJA. A test of missing completely at random for multivariate data with missing values. J Am Stat Assoc. 1988;83(404):1198-1202.

26. Baraldi AN, Enders CK. An introduction to modern missing data analyses. J Sch Psychol. 2010;48(1):5-37.

27. Krause DA, Smith AM, Holmes LC, et al. Relationship of off-ice and on-ice performance measures in high school male hockey players. $J$ Strength Cond Res. 2012;26(5):1423-1430.

28. Hinrichs RN, Cavanagh PR, Williams KR. Upper extremity function in running. I: center of mass and propulsion considerations. Int J Sport Biomech. 1987;3:222-241.

29. Mann R. The Mechanics of Sprinting. Primm, NV: CompuSport; 2005.

30. Young M [webpage on the Internet]. Maximal Velocity Sprint Mechanics. 2006. Available from: http://www.scarboroughtrack.com/sprintingmechanics.pdf. Accessed May 16, 2011.

31. Fortier A, Turcotte RA, Pearsall DJ. Skating mechanics of change-ofdirection maneuvers in ice hockey players. Sports Biomech. 2014;13(4): 341-350.

32. Sheppard JM, Young WB. Agility literature review: classifications, training and testing. J Sports Sci. 2006;24(9):919-932.
Open Access Journal of Sports Medicine

\section{Publish your work in this journal}

The Open Access Journal of Sports Medicine is an international, peer-reviewed, open access journal publishing original research, reports, reviews and commentaries on all areas of sports medicine. The journal is included on PubMed. The manuscript management system is completely online and includes a very quick and fair

\section{Dovepress}

peer-review system. Visit http://www.dovepress.com/testimonials.php to read real quotes from published authors. 